\title{
Communication
}

\section{Effect of Atmospheric Ions on Interfacial Water}

\section{Chien-Chang Kurt Kung ${ }^{1, *}$ and Gerald H. Pollack ${ }^{2}$}

1 Department of Electrical Engineering, Box 352550, University of Washington, Seattle, WA 98195 , USA

2 Department of Bioengineering, Box 355061, University of Washington, Seattle, WA 98195, USA; E-Mail: ghp@uw.edu

* Author to whom correspondence should be addressed; E-Mail: ckung@uw.edu; Tel.: +1-206-407-8945.

External Editor: Kevin H. Knuth

Received: 4 August 2014; in revised form: 30 October 2014 / Accepted: 10 November 2014 / Published: 18 November 2014

\begin{abstract}
The effect of atmospheric positivity on the electrical properties of interfacial water was explored. Interfacial, or exclusion zone (EZ) water was created in the standard way, next to a sheet of Nafion placed horizontally at the bottom of a water-filled chamber. Positive atmospheric ions were created from a high voltage source placed above the chamber. Electrical potential distribution in the interfacial water was measured using microelectrodes. We found that beyond a threshold, the positive ions diminished the magnitude of the negative electrical potential in the interfacial water, sometimes even turning it to positive. Additionally, positive ions produced by an air conditioner were observed to generate similar effects; i.e., the electrical potential shifted in the positive direction but returned to negative when the air conditioner stopped blowing. Sometimes, the effect of the positive ions from the air conditioner was strong enough to destroy the structure of interfacial water by turning the potential decidedly positive. Thus, positive air ions can compromise interfacial water negativity and may explain the known negative impact of positive ions on health.
\end{abstract}

Keywords: interfacial water; atmospheric ions; air ions; air conditioner 


\section{Introduction}

More than 200 years ago, Franklin [1] and Dalibard [2] independently discovered atmospheric electricity. Shortly after, many scientists studied the biological effects of positive and negative air ions [3]. The effects include the killing of bacteria [4], changing the growth rate of plants and insects [5,6], as well as various physiological and behavioral changes in animals, including humans $[7,8]$. However, there is still no agreement to date on the mechanism of how the charged air ions affect biological system.

We discovered a long-range feature of water next to hydrophilic surfaces [9-11] which forms a unique and extensive structure that has different physico-chemical properties from bulk water. This said form water is found to have a net negative charge. This is significant because interfacial water is ubiquitous in nearly all biological environments and involved in all major cellular processes [12], implying that it might be a common target for the known effects of atmospheric electricity. We produced air ions using coronal discharge [13] and studied the effects of those positive air ions on the electrical properties of interfacial water. Clear effects were found. On this basis, we propose a new way to explain the biological effects of air ions.

\section{Materials and Methods}

The electrical properties of interfacial water next to hydrophilic surfaces were measured with and without coronal discharge. Nafion 117 perfluorinated membranes (0.007 in. thick, Sigma-Aldrich, Saint Louis, MO, USA) were used as the hydrophilic surface. Before use, the Nafion membrane was immersed in deionized water for $10 \mathrm{~min}$ at room temperature.

Deionized water was obtained from a NANOpure Diamond ultrapure water system. The purity of water from this system was certified by a resistivity value up to $18.2 \mathrm{M} \Omega$-cm, which exceeded American Society for Testing and Materials (ASTM), College of American Pathologist/ National Committee for Clinical Laboratory Standards (CAP/NCCLS) type I water requirements. In addition, the deionized water was passed through a $0.2-\mu \mathrm{m}$ hollow fiber filter to ensure bacteria- and particle-free water before filling the glass chamber (dimensions: $7 \mathrm{~cm} \times 4.5 \mathrm{~cm} \times 2.5 \mathrm{~cm}$ ) to a consistent height of $1 \mathrm{~cm}$ for all experiments.

As the probing electrode, we used standard glass microelectrodes pulled by a micropipette puller (P-87, Sutter Instrument, Novato, CA, USA) and filled with $3 \mathrm{M} \mathrm{KCl}$. Diameters of the microelectrode tips were less than $1 \mu \mathrm{m}$. To check for electrode quality, the probing and reference electrodes were immersed in $3 \mathrm{M} \mathrm{KCl}$ prior to each experiment. In $3 \mathrm{M} \mathrm{KCl}$, the potential difference between the two electrodes was negligible. However, in deionized water, a DC offset almost always appeared due to deionized water's poor conductivity. The DC offset could be anywhere between $-20 \mathrm{mV}$ to $+20 \mathrm{mV}$. To measure the electrical potential distribution in the water in the vicinity of Nafion, we used a motor to advance the probe electrode. The motor was controlled by software written in Labview, and had a spatial resolution of $1.25 \mu \mathrm{m}$.

A high voltage generator (ES30P-5W, Gamma High Voltage Research) was used to create the coronal discharge, which produced positive air ions.

The experimental setup is shown in Figure 1 Electrical potentials were measured with an electrometer (Model 6512, Keithley Instruments, Cleveland, OH, USA) and recorded on the computer through a data acquisition device (USB-6009, National Instruments, Austin, TX, USA). A flexible reference electrode 
(WPI) was placed remotely from the interfacial region. A $1000 \mathrm{M} \Omega$ passive resistor was placed in parallel with the electrometer terminals to keep the load resistance constant and to facilitate readout of the current extracted from the interfacial water. The resistor also helped to reduce ambient electromagnetic noise.

Figure 1. Experimental setup. (a) The discharge electrodes connected to the high voltage (H.V.) generator; (b) Nafion membrane; (c) The probing microelectrode; (d) $1000 \mathrm{M} \Omega$ passive resistor placed parallel with the electrometer; (e) The reference electrode.

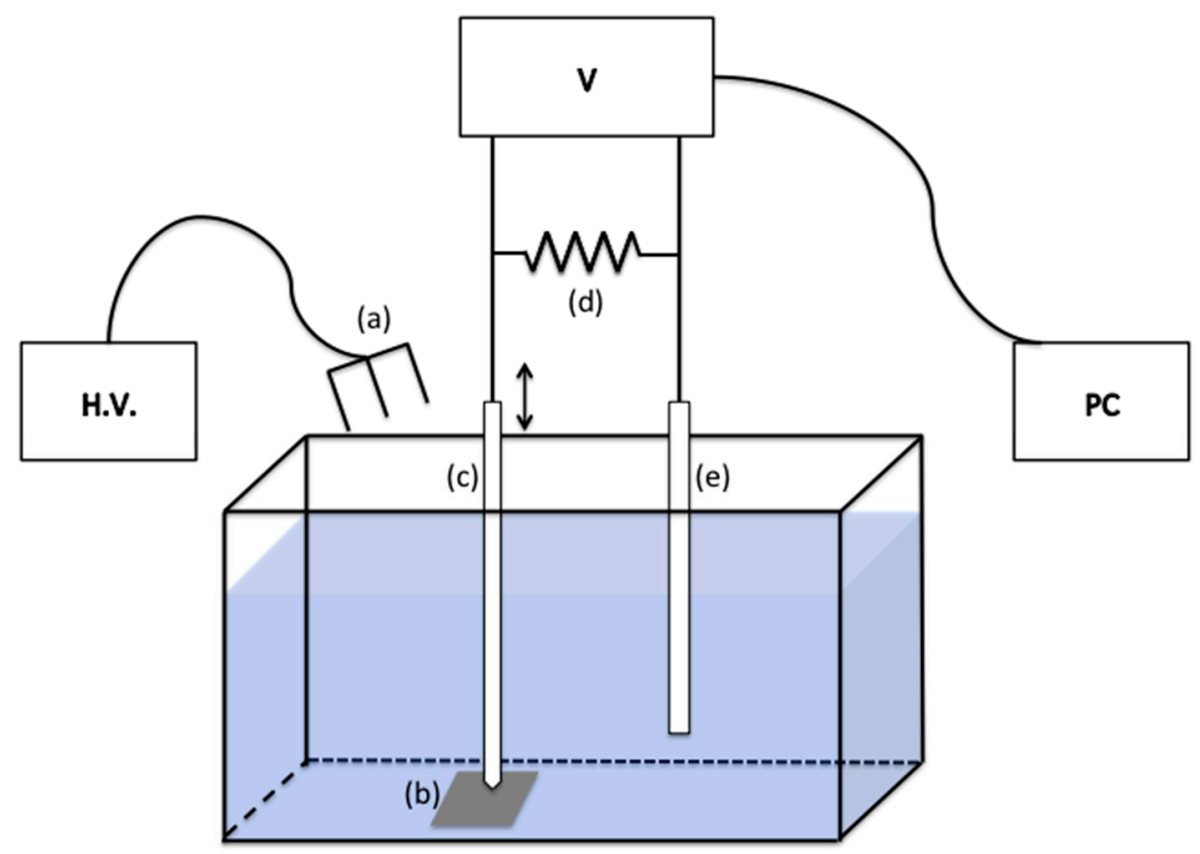

We first positioned the probing microelectrode immediately above and just touching the Nafion surface to obtain the most stable and highest magnitude electrical potential readout. The reference electrode was placed remotely. Before turning on the high voltage source, we measured the potential profile as a function of distance from the Nafion surface. The probing microelectrode was moved away from the Nafion surface by increments of $1.25 \mu \mathrm{m}$ until it reached $1 \mathrm{~mm}$ from the Nafion surface. The electrical potential was recorded at each pause. After the potential profile measurement was completed, the system automatically brought the probing microelectrode back to the Nafion surface. Thus, the data collected provides reference information to be compared with the experimental results obtained in the presence of positive air ions.

High-voltage discharge was used to produce air ions. We turned up the voltage from $0 \mathrm{kV}$ to $10 \mathrm{kV}$ at $1 \mathrm{kV}$ intervals. The discharge electrodes were located $3 \mathrm{~cm}$ above the water surface. Potentials as a function of increasing voltage were measured by the probing electrode and recorded simultaneously. Then we maintained the high voltage at $10 \mathrm{kV}$ for $10 \mathrm{~min}$ and measured the potential profile once again. We set the $10 \mathrm{kV}$ limit because at about 10.5 to $11 \mathrm{kV}$, the discharge electrodes began generating electric arcs which interfered with all nearby electronic equipment and made measurements inconsistent thereafter.

The probing microelectrode was brought back to the Nafion surface automatically after the second potential profile measurement was completed. Then we reversed the procedure by turning down the high 
voltage gradually back to zero and recording the potential between the two electrodes at $1 \mathrm{kV}$ intervals. At the end, the potential profile was recorded again to check the reversibility of the air-ion effect.

Control experiments were conducted to clarify whether the effect on interfacial water was indeed from air ions or from electrical polarization due to the high voltage discharge electrodes or from the ozone generated during the coronal discharge. To test for electrical polarization, we used two identical reference electrodes in deionized water without the Nafion sheet. The two electrodes were placed side by side equally close to the high voltage discharge electrodes horizontally, at different heights in water. To test for the ozone, we used a copper metal mesh to cover the top of the chamber. Since the metal mesh sheet was grounded, no air ions could reach the water, while the electrically neutral ozone could still do so.

\section{Results}

Electrical potential measurements on the water-Nafion interface have been carried out many times with consistent results. When the probing electrode is very close to the Nafion surface, sometimes, even touching, and the reference electrode placed remotely, the magnitude of the potential difference between the two electrodes is the highest. It can range from -100 to $-200 \mathrm{mV}$. Most often, its value is approximately $-120 \mathrm{mV}$.

With the methods described in the Materials and Methods section, we measured the potential profile $v s$. distance from the Nafion surface as well as the highest potential before, during, and after applying the high voltage. Before turning on the high voltage supply, we obtained results similar to those found earlier. A representative potential profile is shown in Figure 2A.

Figure 2. Potential profile as a function of distance from the Nafion surface, to a distance of $1 \mathrm{~mm}$. (A) Measured before turning on the high voltage power supply. (B) Measured ten minutes after turning the high voltage power supply to $10 \mathrm{kV}$. (C) Measured after the high voltage power supply was turned off.
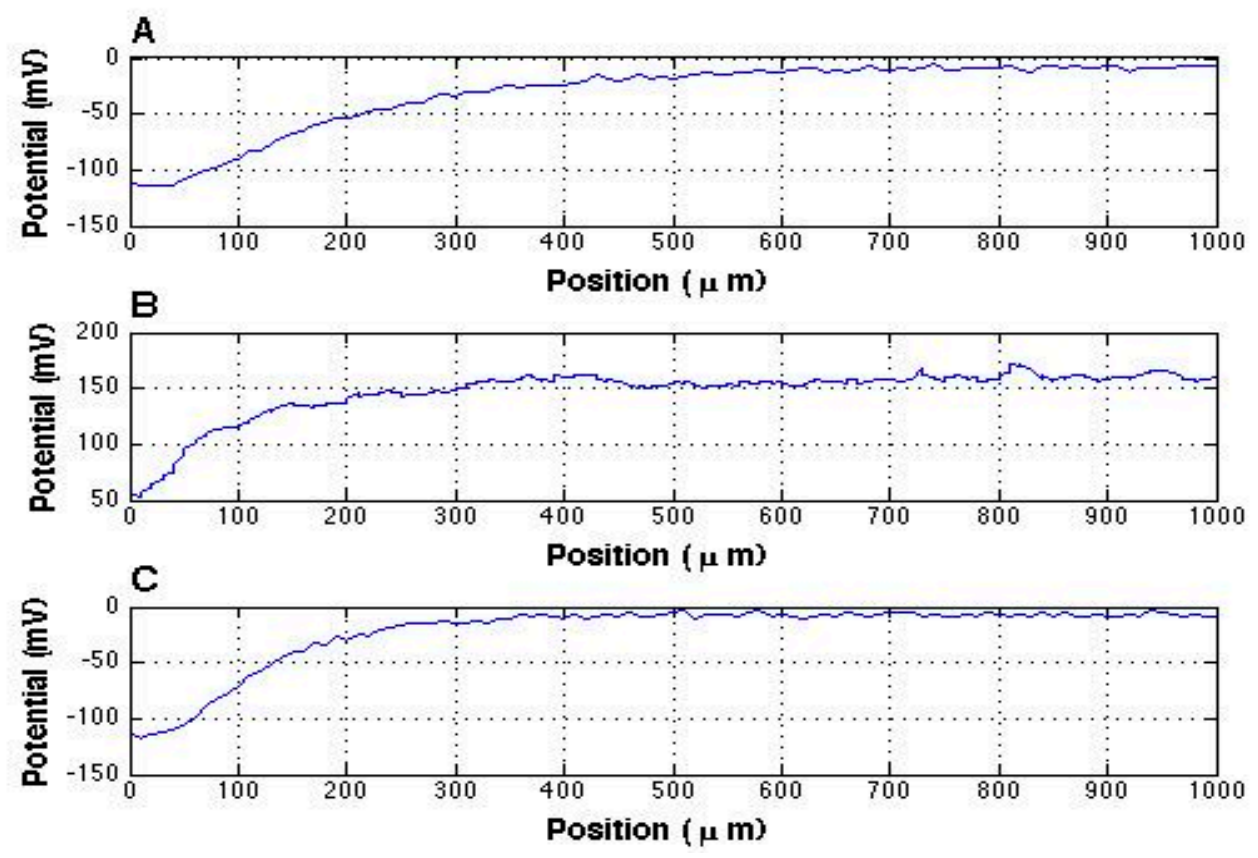
Next, the high voltage power supply was turned on and the potential between the two electrodes was recorded while the voltage was increased in $1 \mathrm{kV}$ increments. This probe electrode remained at a fixed position in the water near the Nafion surface. Figure 3 (blue line) shows that the electrical potential remained the same (less than 3\% change) at all voltages below $8 \mathrm{kV}$. However, starting from around $9 \mathrm{kV}$, the electrical properties of interfacial water began changing noticeably. At the end, even the polarity changed from negative to positive.

Figure 2B depicts the potential profile that was obtained ten minutes after the voltage of the power supply reached $10 \mathrm{kV}$. As can be observed, the potential profile already shifted in the positive zone while the microelectrode was probing in the interfacial water region. This implies that the positive air ions can transfer through to the interfacial water, thus changing its' electrical properties.

The altered electrical properties of the interfacial water were fully reversible. Figure 3 (red line) shows the result obtained during successive reductions of the high voltage. The results fit in nicely with those obtained during the voltage upturn (blue line), except that the order was reversed. The polarity returned to negative between 10 and $9 \mathrm{kV}$, with no obvious change seen from 8 to $0 \mathrm{kV}$. Upon reaching zero, the final potential profile measurement showed a return to the values obtained before turning on the high voltage supply (Figure 2C).

Figure 3. The potential difference between the probe and reference electrodes recorded at progressively increasing (blue)/decreasing (red) increments of $1 \mathrm{kV}$.

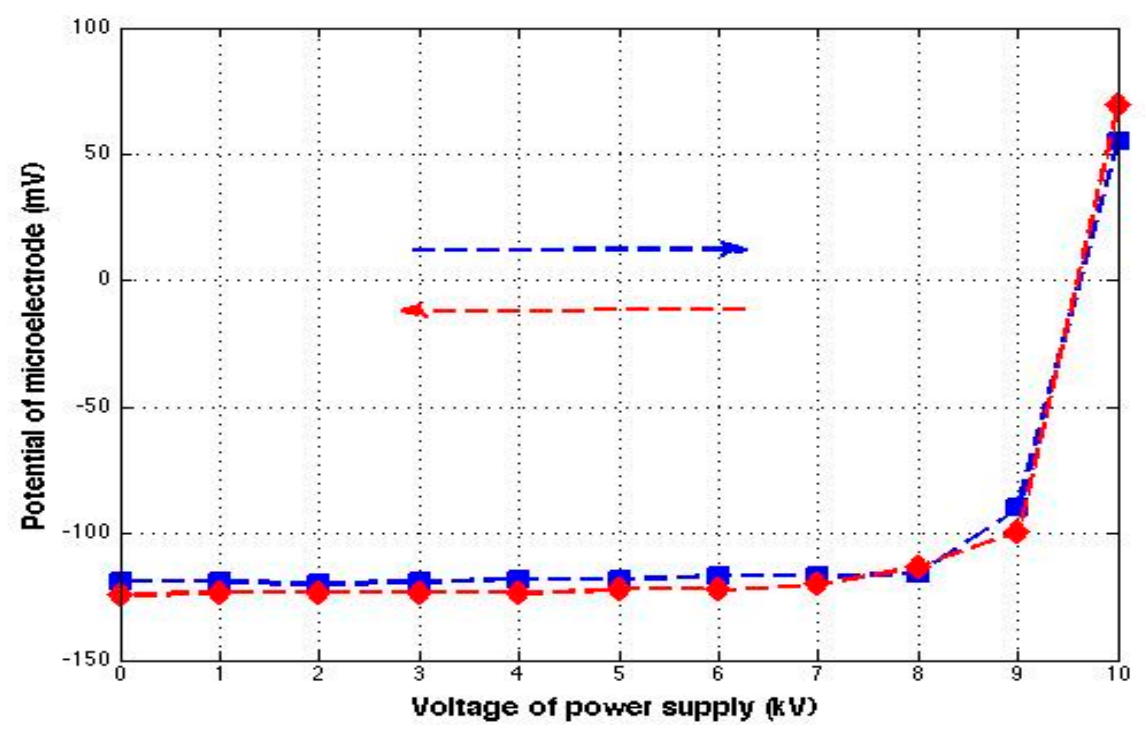

Shutting down the high voltage supply directly from $10 \mathrm{kV}$ produced similar results: the potential between electrodes promptly jumped back approximately to the initial value within just a few seconds. This was an interesting find because positive ions dissolved in water are not expected to neutralize so fast. However, our previous work has shown that interfacial water forms rapidly, implying that the return to negative potential may be due to the rapid re-building of negatively charged interfacial water.

Next, we investigated if the altered electrical properties genuinely arose from the air ions, or from some other features such as polarization of water or ozone produced from coronal discharge.

According to standard electrostatic induction theory, any positive charge placed above a body of water (a dielectric material) should induce negative charge on the top part of the water, while the bottom 
part becomes more positive. However, the air ion effect did the opposite: it made the water more positive on top than the bottom (Figure 2B). Apparently, positive charge from the high voltage source must have entered the water.

To test this idea, we removed the Nafion and placed two identical reference electrodes in the deionized water. One electrode was kept at mid-height of the chamber, while the other was moved vertically, to the top, middle and bottom. When the high voltage source was turned on, the top of the water was clearly more positive than the bottom.

In order to rule out the influence of ozone produced from coronal discharge as the possible cause of the electrical changes, a grounded copper metal mesh was positioned on top of the chamber to block any charged air ions from entering into the water, while allowing the uncharged ozone to pass through. We then elicited coronal discharge by turning up the voltage to $10 \mathrm{kV}$, the highest value possible before creating an electric arc. The potential difference between the two electrodes changed by less than $3 \%$. This observation confirmed that without entry of the charged ions, the electrical properties of interfacial water would not change, implying that the observed potential changes were indeed caused by the positive ions and not by artifacts such as ozone or other byproducts of coronal discharge.

A question is whether naturally occurring changes of atmospheric charge have similar effects on interfacial water. During our earlier experiments on the electrical properties of interfacial water, we noticed small variations in the electrical potential's periodical amplitude. After investigating, we found that those variations were related to the airflow from the laboratory air conditioner: we used an acoustic microphone placed at the air conditioner outlet, and confirmed that the higher the airflow, the louder the noise.

We therefore recorded the airflow over an extended period $(4 \mathrm{~h})$ while simultaneously measuring the electrical potential of interfacial water. We confirmed an excellent alignment of the periodic change in potential (about $4 \mathrm{mV}$ in amplitude) with the airflow from the air conditioner (Figure 4). The period was approximately $50 \mathrm{~min}$. Throughout the experiment, the measured change of water temperature was less than one degree Celsius.

These observations may be explained by the fact that air conditioners are sources of charged air. When air is forced to flow through pipes and filters, it becomes positively charged due to friction. In fact, air is the most positive substance of the triboelectric series [14]. The air's positive charge can help explain how the airflow from the air conditioner can impact the electrical features of interfacial water.

While the effects of the laboratory air conditioner were generally modest, one occasion produced a rather astonishing observation. Figure 5 shows an overnight potential recording with the same set-up as Figure 1 but without the high voltage power supply. The amplitude change was over $400 \mathrm{mV}$, and the period was about $50 \mathrm{~min}$, the same as the air conditioner (compare Figure 4). Because the period is similar, we believe the "skyscraper" oscillations arose from the positively charged air ions coming from the air conditioner.

While there were small periodic changes (similar to those of Figure 4) during the first ten hours of recording, suddenly at about $10.5 \mathrm{~h}$, the potential dramatically changed to a high positive value close to $400 \mathrm{mV}$ (Figure 5A). This kind of threshold effect fits the experimental results described earlier in this paper. We later recorded the potential of interfacial water for another $15 \mathrm{~h}$ without changing the experimental setup and observed similar results (Figure 5B). The one missing skyscraper oscillation near the end of the recording implied that the air conditioner might not have supplied sufficient positive air ions for threshold crossing, the same as during early periods in the two recordings. 
These observations confirmed our findings that airflow from ordinary air conditioners can appreciably change the electrical properties of interfacial water.

Figure 4. Simultaneous recordings of the airflow signal from the air conditioner (A) and the electric potential of interfacial water (B). Note that the peaks and valleys of both the signals are in alignment.
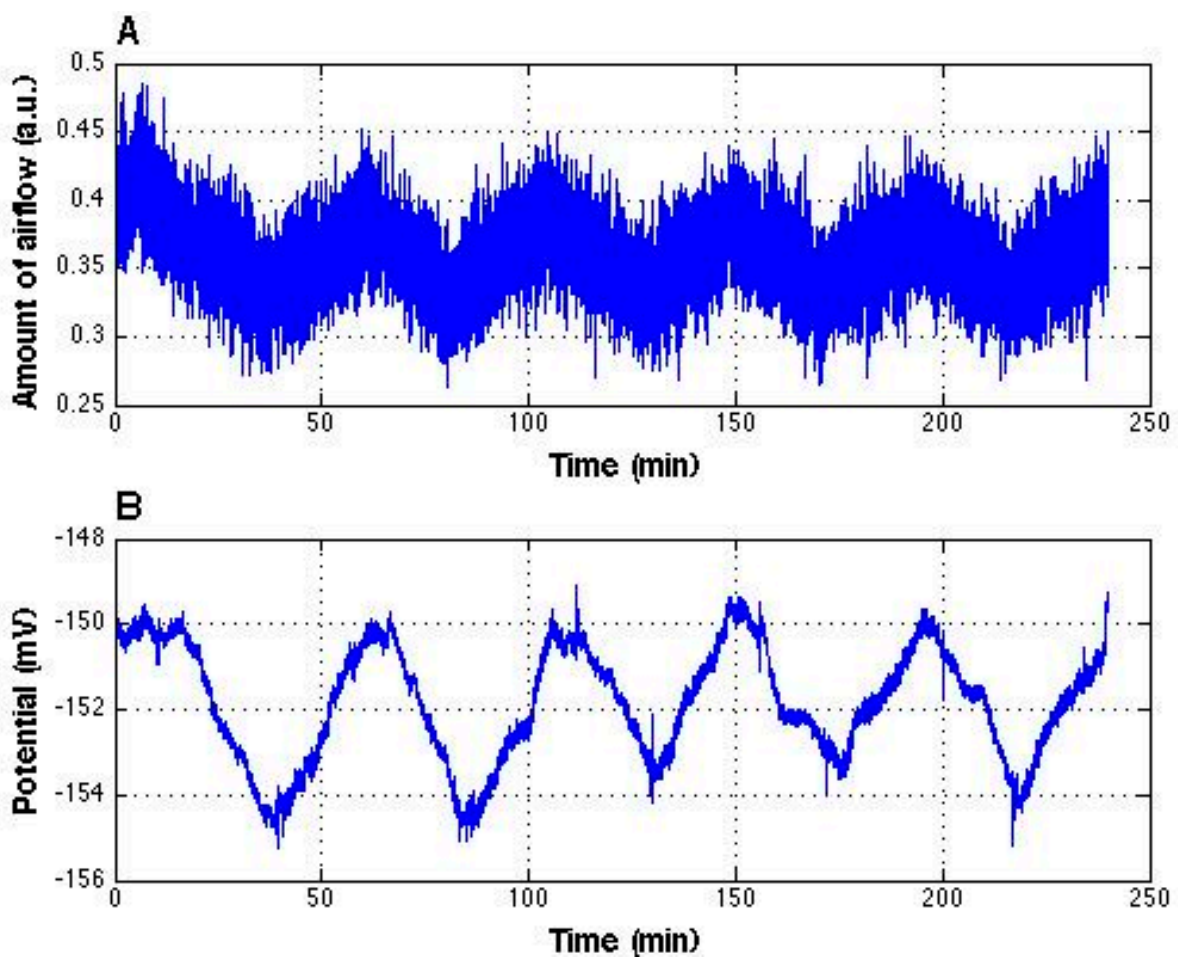

Figure 5. Unusual dramatic change in the overnight potential recording of interfacial water. The first $15 \mathrm{~h}$ (A) were interspersed by an $8 \mathrm{~h}$ gap before the next $15 \mathrm{~h}$ recording (B).
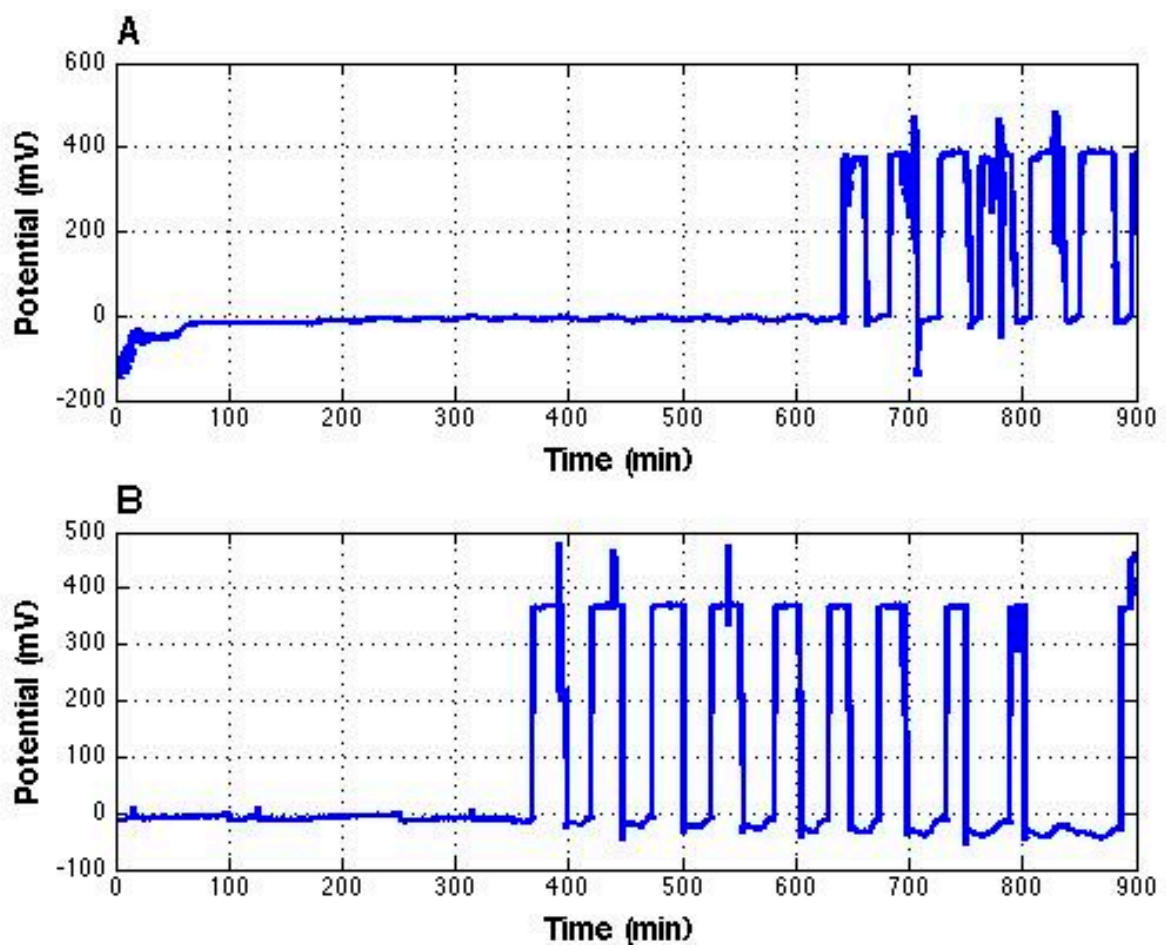


\section{Discussions}

Generally speaking, negative charge is believed to promote good health, whereas positive charge compromises health [7-9,15-17]. Nature provides a reasonably balanced supply of air ions; however more negative ions are found at places such as waterfalls and forests [18], which often elicit sensations of well-being. The question arises whether these effects are largely psychogenic or whether some physical mechanism exists to explain the effects.

One common denominator of function may be cell water. By volume, our bodies are roughly 2/3 of water, and much of that water is interfacial; i.e., it resides next to some hydrophilic interface [12]. More recent experimental observations of interfacial water have shown that interfacial water is negatively charged $[10,11]$. This negative charge could be reduced by atmospheric positivity and thereby cause adverse health effects. In other words, if this water, along with the proteins it envelops, is vital for function, then compromising the amount of interfacial water could impair function.

The experimental results were consistent with this hypothesis. In the experiments involving high voltage, a threshold voltage was required to produce enough air ions to have appreciable impact on the interfacial water. When the discharge electrodes were placed $3 \mathrm{~cm}$ from the water surface, the threshold voltage required to produce enough air ions was approximately $8 \mathrm{kV}$. From this result we infer that positive ions in the air may change concentration appreciably without having adverse health effects. However, beyond a threshold concentration, the ill effects of positive charge may become appreciableeven wiping out the negativity of interfacial water and replacing it with the many positive ions in the water.

The rapid rebuilding of interfacial water once the positive ions were removed is consistent with earlier results. Interfacial water builds rapidly. Once the inhibiting positive ions are removed, the structure can easily and quickly rebuild. In terms of health impact, this suggests that restoration of function can occur rapidly once the positive air ions are removed. This phenomenon can potentially explain the "refreshing" sensation that people often feel when stepping out of a crowded room with positive ions from exhalation, and into fresh air.

We obtained similar results with positive ions produced from an air conditioner. When the conditions are right, enough air ions could be produced to change the electrical properties of the interfacial water. The example of dramatically changed electrical potential indicated that the structure of interfacial water could be completed destroyed by positive air ions, perhaps explaining the serious consequences people can sometimes suffer in sufficiently foul air. More generally, this result may explain the natural craving to escape from air-conditioned environments and into natural air.

\section{Author Contributions}

Kurt Kung and Gerald Pollack designed this research; Kurt Kung performed research and analyzed data; Kurt Kung and Gerald Pollack wrote this paper. Both authors have read and approved the final manuscript.

\section{Conflicts of Interest}

The authors declare no conflict of interest. 


\section{References}

1. Franklin, B.A. Letter from Mr. Franklin to Mr. Peter Collinson, F.R.S. concerning the effects of lightning. Philos. Trans. R. Soc. Lond. 1751, 47, 289-291.

2. Dalibard, T.F. Letter to Academy of Science. 1749.

3. Krueger, A.P. The biological effects of air ions. Int. J. Biometeorol. 1985, 29, 205-206.

4. Phillips, G.; Harris, G.J.; Jones, M.W. Effect of air ions on bacterial aerosols. Int. J. Biometeorol. 1964, 8, 27-37.

5. Krueger, A.P. Studies on the Effects of Gaseous Ions on Plant Growth: I. The influence of positive and negative air ions on the growth of Avena sativa. J. Gen. Physiol. 1962, 45, 879-895.

6. Diamond, M.; Connor, J.; Orenberg, E.; Bissell, M.; Yost, M.; Krueger, A. Environmental influences on serotonin and cyclic nucleotides in rat cerebral cortex. Science 1980, 210, 652-654.

7. Palti, Y.; de Nour, E.; Abrahamov, A. the effect of atmospheric ions on the respiratory system of infants. Pediatrics 1966, 38, 405-411.

8. Kotaka, S.; Krueger, A.P. Effects of air ions on microorganisms and other biological materials. Crit. Rev. Microbiol. 1978, 6, 109-150.

9. Charry, J.M.; Hawkinshire, F.B. Effects of atmospheric electricity on some substrates of disordered social behavior. 1981, 41, 185-197.

10. Zheng, J.-M.; Chin, W.-C.; Khijniak, E.; Khijniak, E., Jr.; Pollack, G.H. Surfaces and interfacial water: Evidence that hydrophilic surfaces have long-range impact. Adv. Colloid Interface Sci. 2006, 127, 19-27.

11. Pollack, G.H. The Fourth Phase of Water: Beyond Solid, Liquid, and Vapor; Ebner and Sons Publishers: Seattle, WA, USA, 2013.

12. Pollack, G.H. Cells, Gels and the Engines of Life; Ebner and Sons Publishers: Seattle, WA, USA, 2001.

13. Hawley, R. Electrical Coronas; University of California Press: Berkeley, CA, USA, 1965.

14. Adams, C.K. Nature's Electricity; TAB Books: Blue Ridge Summit, PA, USA, 1987.

15. Bachman, C.H.; McDonald, R.D.; Lorenz, P.J. Some physiological effects of measured air ions. Int. J. Biometeorol. 1965, 9, 127-139.

16. SovijÄrvi, A.R.A.; Rosset, S.; HyvÄrinen, J.; Franssila, A.; Graeffe, G.; LehtimÄki, M. Effect of air ionization on heart rate and perceived exertion during a bicycle exercise test. Eur. J. Appl. Physiol. Occup. Physiol. 1979, 41, 285-291.

17. Charry, J.M. Biological effects of small air ions: A review of findings and methods. Environ. Res. 1984, 34, 351-389.

18. Luts, A.; Parts, T.E.; Laakso, L.; Hirsikko, A.; Gronholm, T.; Kulmala, M. Some air electricity phenomena caused by waterfalls: Correlative study of the spectra. Atmos. Res. 2009, 91, 229-237.

(C) 2014 by the authors; licensee MDPI, Basel, Switzerland. This article is an open access article distributed under the terms and conditions of the Creative Commons Attribution license (http://creativecommons.org/licenses/by/4.0/). 